\title{
Title: Cutibacterium acnes antibiotic production shapes niche competition in the human skin microbiome
}

Authors: Jan Claesen ${ }^{1}$, Jennifer B Spagnolo ${ }^{2,3 \#}$, Stephany Flores Ramos ${ }^{2 \#}$, Kenji L Kurita ${ }^{4}$, Allyson L Byrd ${ }^{5}$, Alexander A Aksenov ${ }^{6}$, Alexey V Melnik ${ }^{6}$, Weng R Wong ${ }^{7}$, Shuo Wang ${ }^{9}$, Ryan D Hernandez ${ }^{7,8}$, Mohamed S Donia ${ }^{10}$, Pieter C Dorrestein ${ }^{6}$, Heidi H Kong ${ }^{11}$, Julia A Segre $^{5}$, Roger G Linington ${ }^{4}$, Michael A Fischbach ${ }^{12 * \dagger}$ and Katherine P Lemon ${ }^{2,13 * \dagger}$

\section{Affiliations:}

${ }^{1}$ Department of Cardiovascular and Metabolic Sciences, Lerner Research Institute, Cleveland Clinic, Cleveland, OH, USA

${ }^{2}$ Department of Microbiology, The Forsyth Institute, Cambridge, MA, USA.

${ }^{3}$ Department of Oral Medicine, Infection \& Immunity, Harvard School of Dental Medicine, Boston, Massachusetts, USA

${ }^{4}$ Department of Chemistry, Simon Fraser University, Burnaby, BC, Canada

${ }^{5}$ Microbial Genomics Section, National Human Genome Research Institute, National Institutes of Health, Bethesda, MD, USA

${ }^{6}$ Collaborative Mass Spectrometry Innovation Center, Skaggs School of Pharmacy and Pharmaceutical Sciences, and Center for Microbiome Innovation, University of California, San Diego, La Jolla, USA

${ }^{7}$ Department of Bioengineering and Therapeutic Sciences, University of California, San Francisco, San Francisco, CA, USA

${ }^{8}$ Department of Human Genetics, McGill University and Genome Quebec Innovation Center, Montreal, QC, Canada

${ }^{9}$ Department of Chemical and Biological Engineering, Princeton University, Princeton, NJ, USA

${ }^{10}$ Department of Molecular Biology, Princeton University, Princeton, NJ, USA

${ }^{11}$ Dermatology Branch, National Institute of Arthritis and Musculoskeletal and Skin Diseases, National Institutes of Health, Bethesda, MD, USA

${ }^{12}$ Department of Bioengineering and ChEM-H, Stanford University, Stanford, CA, USA

${ }^{13}$ Division of Infectious Diseases, Boston Children's Hospital and Harvard Medical School, Boston, MA, USA

$30 \quad$ *Correspondence to: fischbach@ fischbachgroup.org and klemon@ forsyth.org;

Katherine.lemon@ childrens.harvard.edu

${ }^{\text {\#Equal contribution }}$

${ }^{\dagger}$ Equal contribution 
Abstract: The composition of the skin microbiome varies widely among individuals sampled at the same body site. A key question is which molecular factors determine strain-level variability within sub-ecosystems of the skin. We used a genomics-guided approach to identify an antibacterial biosynthetic gene cluster in Cutibacterium acnes (formerly Propionibacterium acnes) that is widely distributed across individuals and skin sites. Experimental characterization of this cluster enabled the identification of a new thiopeptide antibiotic, cutimycin. Analysis of individual human skin hair follicles showed that cutimycin is an important factor regulating colonization resistance against Staphylococcus species.

One Sentence Summary: Cutimycin, a thiopeptide antibiotic produced by a widespread skin commensal, reduces Staphylococcus colonization of human follicles.

\section{Main Text:}

Niche competition among resident microbiota is postulated to influence skin microbial community composition via colonization resistance. In conjunction with host environmental factors (e.g., desiccation, low pH, high salt and high lipid concentrations (1)) and the host immune response (2), this results in a distinctive skin microbiota with variations among sites that are characterized as being predominantly sebaceous, moist or dry. Species of Staphylococcus, Corynebacterium and Cutibacterium (formerly the cutaneous Propionibacterium (3)) are among the most prevalent and abundant members of the human skin microbiota.

Recent studies have begun to uncover mechanisms of competition among bacterial species that shape microbiota composition across human skin (4). Small molecules are one means by which bacteria interact with each other and their environment. The genes required to produce these small molecules co-localize in biosynthetic gene clusters (BGCs) (5). BGCs are abundant in the human microbiome (6), but relatively few have a proven function (6-8). For example, the nasal and skin colonizer Staphylococcus lugdunensis produces a nonribosomal peptide, lugdunin, that inhibits growth of and colonization by $S$. aureus (7). Similarly, some strains of coagulasenegative Staphylococcus produce antimicrobial peptides that kill S. aureus. The levels of these strains are reduced in atopic dermatitis when $S$. aureus predominates and their expansion decreases skin colonization by S. aureus (8). Other mechanisms of bacterial competition on skin and in the nostrils include protease activity (9), disruption of Staphylococcus quorum sensing $(10,11)$, competition for iron (12), bacterial release of antimicrobial free fatty acids from host triacylglycerols (13), and niche competition mediated by the host (14). Nonetheless, the examples of lugdunin (7) and Staphylococcus-derived lanthipeptides (8) highlight the key roles of BGCs and their products for members of the human microbiota, and the need to identify and characterize secreted antibacterial compounds from other members of human skin and nasal microbiota $(15,16)$.

One widely distributed family of BGCs in the human microbiome is predicted to encode a subclass of ribosomally synthesized, post-translationally modified peptides (RiPPs) known as thiopeptides (6). Many thiopeptides, such as berninamycin from Streptomyces bernensis and the semi-synthetic LFF571, which was used in a Phase 2 clinical study, have potent antistaphylococcal activity via inhibition of protein synthesis $(17,18)$. We noted that the most widely distributed thiopeptide BGC in the human microbiome is found in 8/219 (3.7\%) sequenced isolates of Cutibacterium species (Table S1), all Cutibacterium acnes. We and others predict that the product of this BGC (ppa0859-0866 in C. acnes strain KPA171202; Fig. 1A) is a thiopeptide $(19,20)$ structurally related to the antibiotic berninamycin $(21,22)$. 
Cutibacterium species are particularly well adapted to life on human skin with their ability to thrive in the lipid-rich environment of the human skin hair follicle, with its associated sebaceous gland. Hypothesizing that the small molecule product of this predicted cutimycin BGC plays a role in skin microbial community composition, we set out to identify this compound and determine its structure.

Based on its computationally predicted similarity to berninamycin (23), we hypothesized that the Cutibacterium BGC encodes a thiopeptide that $C$. acnes uses to target Staphylococcus species (phylum Firmicutes) in their shared habitats on human skin, including the skin inside the nostrils. To test for production of a thiopeptide, we selected phylogenetically distinct $C$. acnes strains that differ with respect to the presence or absence of BGCs. By analyzing crude culture extracts from these strains by HPLC, we observed that the thiopeptide BGC+ isolate $C$. acnes HL030PA1 produces a compound with a retention time and UV absorption spectrum similar to that of the thiopeptide berninamycin (Fig. 1B, blue trace).

To determine whether the BGC from $C$. acnes HL030PA1 was sufficient to produce the observed compound, we expressed this cluster heterologously in a related Actinobacterium, Corynebacterium glutamicum. We analyzed organic extracts prepared from cell pellets of the wild-type and BGC+ strains of $C$. glutamicum, observing that the latter but not the former contained a molecule identical to the one produced by $C$. acnes HL030PA1 (Fig. 1C). Unlike in the cell pellet, we did not detect the molecule in an extract of the culture supernatant of the $\mathrm{BGC}+C$. glutamicum. These data establish that the Cutibacterium BGC is sufficient for the biosynthesis of the molecule detected, but not its export.

Because of higher production yields and the ability to grow under aerobic conditions, we scaled up cultivation of the cutimycin BGC+C. glutamicum and purified the thiopeptide (Fig. S1). Determination of the accurate mass at $\mathrm{m} / \mathrm{z} 1131.3364$ allowed prediction of a formula of C51H51N14O15S + , with a predicted monoisotopic mass of $1131.3373\left(\Delta_{\text {theoretical }}=0.8 \mathrm{ppm}\right)$ (Figs. S2 \& S3). The planar structure of the thiopeptide was solved de novo based on 1D and 2D NMR experiments and HRMS ${ }^{\mathrm{e}}$ and is described in depth in the supplemental information (Figs. S4-S11; Table S2). The configuration of each stereogenic center was determined using Marfey's analysis (Fig. S12). We defined atom position numbering and the amino acid numbering (Figs. $1 \mathrm{D}$ and S13) following the convention set in the previous publication of the structure of berninamycin for ease of comparison (24). We assigned the trivial name cutimycin to this Cutibacterium-derived thiopeptide.

Cutimycin has potent activity in vitro against Staphylococcus but not against commensal Actinobacteria from skin. Based on cutimycin's structural similarity to berninamycin and LFF571 (Fig. S14), we hypothesized it would also display anti-staphylococcal activity but would lack activity against common Actinobacteria skin commensals. To test this, we determined the MICs for cutimycin and berninamycin against a selection of species commonly found on skin sites, including the nostrils (Table S3). Cutimycin exhibited potent inhibition of the USA300 community associated methicillin-resistant $S$. aureus strain NRS384 (MIC $0.2 \mu \mathrm{M}$ ), as well as strains of Staphylococcus epidermidis. In contrast, a panel of other $C$. acnes strains, with and without the cutimycin BGC, plus two common skin and two common nasal Corynebacterium species (phylum Actinobacteria) displayed increased resistance to cutimycin with MICs $\geq 3.2$ $\mu \mathrm{M}$. These data led us to hypothesize that cutimycin favors the growth of resident skin Actinobacteria, including C. acnes, over that of common skin staphylococcal species. 
Cocultivation with susceptible Staphylococcus strains increased transcription of the cutimycin BGC. The production of secondary metabolites can be costly and BGCs often contain regulatory mechanisms for inducible rather than constitutive expression. We did not identify an obvious regulatory element in the cutimycin BGC. However, we hypothesized that cutimycin-susceptible species would induce transcription of cutimycin, whereas resistant species would not. To test this, we assayed transcription of the cutimycin BGC during in vitro cocultivation of $C$. acnes with $S$. aureus, $S$. epidermidis (both susceptible) or Corynebacterium striatum (resistant) compared to $C$. acnes monocultivation. For this qRT-PCR-based assay, we used C. acnes KPA171202 because the encoded cutimycin BGC (25) is transcribed during exponential growth in vitro (19). Compared to when $C$. acnes was grown alone, transcript levels of ppa0860 from the cutimycin BGC in C. acnes KPA171202 increased twofold in the presence of $S$. aureus or $S$. epidermidis, but decreased in presence of $C$. striatum (0.16 fold) (Fig. 2). These results indicate that cutimycin transcription is selectively increased by the presence of Staphylococcus targets. To determine whether cutimycin is produced in vivo in C. acnes' natural habitat, we used mass spectrometry to analyze pooled content from hair follicles of intact skin of individual adult volunteers. Due to the small quantity of each sample (25-80 follicles), it was not possible to observe cutimycin using untargeted mass spectrometry (26). Using targeted mass spectrometry, we detected cutimycin in $28 \%$ of the samples (Fig. S15, Table S4). Based on an external standard and a typical hair follicle volume of $0.2 \mathrm{~mm}^{3}(27)$, the concentration of cutimycin was estimated to be to be $0.97+/-0.12 \mu \mathrm{M}$ in the samples where the molecule was detected. However, the local concentrations likely exceed this value as cutimycin-producing strains are unlikely to be evenly distributed throughout the follicle. Based on these data, we hypothesized that cutimycin plays a role in modulating levels of Staphylococcus in the context of human skin colonization.

The cutimycin BGC is widely distributed on human skin but present in only a subset of strains at each body site. Humans harbor an abundance of Cutibacterium on their skin, with $C$. acnes being the overwhelmingly predominant species. In the absence of an animal model for $C$. acnes colonization of skin hair follicles, we began by examining isolate and metagenomic sequence data to explore a potential role for the cutimycin BGC in modulating the composition of the human skin community. The cutimycin BGC is present in the genomes of only about 4\% (8/219 in Table S1) of sequenced $C$. acnes isolates. However, in a longitudinal high-resolution skin metagenomic dataset from 12 healthy volunteers (28), the cutimycin BGC could be detected in $11 / 12$ individuals. When it was present in an individual, it could be identified across sampled sites stably over time (orange bars in Fig. 3A and S16). In spite of its broad distribution, in the majority of samples $<30 \%$ of the total $C$. acnes contained the BGC. C. acnes is also a common member of the human nostril microbiome, which is assayed from the skin of the nasal vestibule $(29,30)$. Because this site was absent in our metagenomic dataset, we also assayed for the cutimycin BGC in nostril metagenomic data from the Human Microbiome Project, finding it in $8 / 75$ samples (10.7\%). From the 12 intensively sampled volunteers, we also examined the 40 distribution of four other $C$. acnes BGCs predicted to code for interesting bioactive compounds (Table S5). Similar to the cutimycin BGC, we observed a wide distribution with strain-level variation for these other Cutibacterium BGCs on adult human skin (Figs. 3A and S16). There were, however, no significant correlations between the absence/presence of the cutimycin BGC, or any other predicted $C$. acnes BGC, and the abundance of specific skin commensals in these 12 volunteers. At first these results seemed incongruous with cutimycin's anti-staphylococcal activity. However, we reasoned that the spatial scale of a skin swab is much larger than the scale 
at which $C$. acnes interacts with neighboring bacterial species. Therefore, we hypothesized that a much finer spatial resolution was required to test whether cutimycin has an effect on microbial community composition. Because $C$. acnes is a known resident of human skin follicles, we next assayed the distribution of the cutimycin BGC and its potential impact on community composition at the level of individual human skin hair follicles.

Skin follicles containing the cutimycin BGC have a higher ratio of $C$. acnes to $S$. epidermidis. To address the distribution question across individual skin hair follicles, we sampled the contents of 6-10 healthy follicles from the outer surface of the nose of 16 human volunteers (Fig. S17A, B and Table S6). For each follicle, we quantified colony forming units (CFUs) to assay community composition and performed PCR of Cutibacterium isolates to determine the presence or absence of the cutimycin BGC. From 156 individual follicles, we identified three bacterial species by $16 \mathrm{~S}$ rRNA gene sequencing of representative colonies: $C$. acnes, Cutibacterium granulosum, and $S$. epidermidis. These three species were easily distinguished by colony morphology (Fig. S17C), permitting quantification of each in follicular content. Since follicles differed in the total number of CFUs (possibly due to size, sampling or true variation), we measured the ratio of $C$. acnes/S. epidermidis in follicles with and without the cutimycin BGC (Fig. S17D-E). Strikingly, follicles that contained $C$. acnes strains that were cutimycin BGC-positive had higher $C$. acnes/S. epidermidis ratio than those that were cutimycin BGC negative (Wilcoxon signed-rank test, $p=0.006$ ) (Fig. 3B). This finding suggests a role for cutimycin in modulating the composition of the skin microbiome, and it highlights that the characteristic spatial scale of this interaction is tiny - that of an individual follicle.

We also observed some cutimycin BGC-negative follicles with a high C. acnes/S. epidermidis ratio. One possible explanation is that alternative $C$. acnes-produced anti-Staphylococcus activities are present within these follicles, possibly encoded by one of the other predicted $C$. acnes BGCs (Fig. 3A and S16). However, other bacterial molecules/mechanisms could be mediating the competitive interactions, e.g., nutrient competition, toxic primary metabolites (31) or antimicrobial free fatty acids released from host triacylglycerols (32), as well as possible hostmediated effects.

In addition to inferences regarding the impact of cutimycin on community composition, these data on the presence or absence of the cutimycin BGC in individual human skin follicles indicate that follicles might be colonized by at least 2 different $C$. acnes strains and that $C$. acnes strainlevel colonization is punctate, sometimes varying from follicle to follicle in an individual (Fig. S17D; Table S6). Thus, future exploration of the effect of $C$. acnes small molecules on human skin microbiota requires investigating at the fine-scale resolution of the individual skin follicle (Fig. S17C; Table S6).

Here, we have identified a molecular mechanism of niche competition between two of the most common members of the human skin microbiome: C. acnes and Staphylococcus species. With this work, cutimycin becomes one of the few BGCs from the human skin microbiome with a known molecular function. Our elucidation of cutimycin's function will facilitate exploration into possible clinical applications of cutimycin to selectively inhibit Staphylococcus colonization, while leaving commensal Actinobacteria undisturbed. S. aureus nasal colonization is a risk for invasive infection (33) and, in the absence of an effective anti-staphylococcal vaccine (34), there is a need to identify strains of beneficial bacteria and their bioactive products that could be used to generate nasal and skin microbiota resistant to colonization by $S$. aureus. Such approaches might also have application in preventing or treating skin diseases that include 
a shift in either microbiome composition, such as in atopic dermatitis flares, or in host-microbe interactions, such as acne vulgaris $(35,36)$.

In conclusion, this successful elucidation of the cutimycin-mediated competition of $C$. acnes with Staphylococcus in vivo on humans demonstrates the power of combining systems-level approaches (e.g., in silico mining to characterize an antibiotic from the skin microbiome) with reductionist approaches (e.g., the in vitro cultivation of known bacterial colonizers of human skin follicles to explore of the impact of cutimycin at the fine-scale resolution of the individual skin follicle) to discover new mechanisms that underlie the ecology of the microbiome.

\section{References and Notes:}

1. Y. E. Chen, M. A. Fischbach, Y. Belkaid, Skin microbiota-host interactions. Nature 553, 427-436 (2018).

2. A. L. Byrd, Y. Belkaid, J. A. Segre, The human skin microbiome. Nat Rev Microbiol 16, 143-155 (2018).

3. C. F. Scholz, M. Kilian, The natural history of cutaneous propionibacteria, and reclassification of selected species within the genus Propionibacterium to the proposed novel genera Acidipropionibacterium gen. nov., Cutibacterium gen. nov. and Pseudopropionibacterium gen. nov. Int J Syst Evol Microbiol 66, 4422-4432 (2016).

4. A. L. Cogen, V. Nizet, R. L. Gallo, Skin microbiota: a source of disease or defence? $B r J$ Dermatol 158, 442-455 (2008).

5. P. Cimermancic et al., Insights into secondary metabolism from a global analysis of prokaryotic biosynthetic gene clusters. Cell 158, 412-421 (2014).

6. M. S. Donia et al., A systematic analysis of biosynthetic gene clusters in the human microbiome reveals a common family of antibiotics. Cell 158, 1402-1414 (2014).

7. A. Zipperer et al., Human commensals producing a novel antibiotic impair pathogen colonization. Nature 535, 511-516 (2016).

8. T. Nakatsuji et al., Antimicrobials from human skin commensal bacteria protect against Staphylococcus aureus and are deficient in atopic dermatitis. Sci Transl Med 9, (2017).

9. T. Iwase et al., Staphylococcus epidermidis Esp inhibits Staphylococcus aureus biofilm formation and nasal colonization. Nature 465, 346-349 (2010).

10. A. E. Paharik et al., Coagulase-Negative Staphylococcal Strain Prevents Staphylococcus aureus Colonization and Skin Infection by Blocking Quorum Sensing. Cell Host Microbe 22, 746-756 e 745 (2017).

11. M. M. Ramsey, M. O. Freire, R. A. Gabrilska, K. P. Rumbaugh, K. P. Lemon, Staphylococcus aureus Shifts toward Commensalism in Response to Corynebacterium Species. Front Microbiol 7, 1230 (2016).

12. R. M. Stubbendieck et al., Competition among nasal bacteria suggests a role for siderophore-mediated interactions in shaping the human nasal microbiota. Appl Environ Microbiol, (2018). 
13. L. Bomar, S. D. Brugger, B. H. Yost, S. S. Davies, K. P. Lemon, Corynebacterium accolens Releases Antipneumococcal Free Fatty Acids from Human Nostril and Skin Surface Triacylglycerols. MBio 7, e01725-01715 (2016).

14. M. Otto, Staphylococcus colonization of the skin and antimicrobial peptides. Expert Rev Dermatol 5, 183-195 (2010).

15. D. Janek, A. Zipperer, A. Kulik, B. Krismer, A. Peschel, High Frequency and Diversity of Antimicrobial Activities Produced by Nasal Staphylococcus Strains against Bacterial Competitors. PLoS Pathog 12, e1005812 (2016).

16. J. N. O'Sullivan, M. C. Rea, P. M. O'Connor, C. Hill, R. P. Ross, Human skin microbiota is a rich source of bacteriocin-producing staphylococci that kill human pathogens. FEMS Microbiol Ecol 95, (2019).

17. D. M. Citron, K. L. Tyrrell, C. V. Merriam, E. J. Goldstein, Comparative in vitro activities of LFF571 against Clostridium difficile and 630 other intestinal strains of aerobic and anaerobic bacteria. Antimicrob Agents Chemother 56, 2493-2503 (2012).

18. K. Mullane et al., Multicenter, randomized clinical trial to compare the safety and efficacy of LFF571 and vancomycin for Clostridium difficile infections. Antimicrob Agents Chemother 59, 1435-1440 (2015).

19. E. Brzuszkiewicz et al., Comparative genomics and transcriptomics of Propionibacterium acnes. PLoS One 6, e21581 (2011).

20. L. C. Wieland Brown, M. G. Acker, J. Clardy, C. T. Walsh, M. A. Fischbach, Thirteen posttranslational modifications convert a 14-residue peptide into the antibiotic thiocillin. Proc Natl Acad Sci U S A 106, 2549-2553 (2009).

21. H. Abe, K. Kushida, Y. Shiobara, M. Kodama, The structures of sulfomycin I and berninamycin A. Tetrahedron Lett 29, 1401-1404 (1988).

22. R. C. Lau, K. L. Rinehart, Berninamycins B, C, and D, minor metabolites from Streptomyces bernensis. J Antibiot (Tokyo) 47, 1466-1472 (1994).

23. M. E. Bergy, J. H. Coats, F. Reusser, Antibiotic berninamycin and process for making same. US3689639 A. (1972).

24. S. J. Malcolmson, T. S. Young, J. G. Ruby, P. Skewes-Cox, C. T. Walsh, The posttranslational modification cascade to the thiopeptide berninamycin generates linear forms and altered macrocyclic scaffolds. Proc Natl Acad Sci U S A 110, 8483-8488 (2013).

25. H. Brüggemann et al., The complete genome sequence of Propionibacterium acnes, a commensal of human skin. Science 305, 671-673 (2004).

26. A. V. Melnik et al., Coupling Targeted and Untargeted Mass Spectrometry for Metabolome-Microbiome-Wide Association Studies of Human Fecal Samples. Anal Chem 89, 7549-7559 (2017).

27. N. Otberg et al., Variations of hair follicle size and distribution in different body sites. $J$ Invest Dermatol 122, 14-19 (2004). 
28. J. Oh et al., Temporal Stability of the Human Skin Microbiome. Cell 165, 854-866 (2016).

29. C. Human Microbiome Project, Structure, function and diversity of the healthy human microbiome. Nature 486, 207-214 (2012).

30. I. F. Escapa et al., New Insights into Human Nostril Microbiome from the Expanded Human Oral Microbiome Database (eHOMD): a Resource for the Microbiome of the Human Aerodigestive Tract. mSystems 3, (2018).

31. M. Shu et al., Fermentation of Propionibacterium acnes, a commensal bacterium in the human skin microbiome, as skin probiotics against methicillin-resistant Staphylococcus aureus. PLoS One 8, e55380 (2013).

32. R. R. Marples, D. T. Downing, A. M. Kligman, Control of free fatty acids in human surface lipids by Corynebacterium acnes. J Invest Dermatol 56, 127-131 (1971).

33. C. von Eiff, K. Becker, K. Machka, H. Stammer, G. Peters, Nasal carriage as a source of Staphylococcus aureus bacteremia. N Engl J Med 344, 11-16 (2001).

34. B. K. Giersing, S. S. Dastgheyb, K. Modjarrad, V. Moorthy, Status of vaccine research and development of vaccines for Staphylococcus aureus. Vaccine 34, 2962-2966 (2016).

35. A. M. O'Neill, R. L. Gallo, Host-microbiome interactions and recent progress into understanding the biology of acne vulgaris. Microbiome 6, 177 (2018).

36. S. Fitz-Gibbon et al., Propionibacterium acnes strain populations in the human skin microbiome associated with acne. J Invest Dermatol 133, 2152-2160 (2013).

37. D. G. Gibson et al., Enzymatic assembly of DNA molecules up to several hundred kilobases. Nat Methods 6, 343-345 (2009).

38. L. Eggeling, M. Bott, Handbook of Corynebacterium glutamicum. (Taylor \& Francis, Boca Raton, 2005), pp. 616 p.

39. A. Zhu, S. Sunagawa, D. R. Mende, P. Bork, Inter-individual differences in the gene content of human gut bacterial species. Genome Biol 16, 82 (2015).

40. H. Li, A statistical framework for SNP calling, mutation discovery, association mapping and population genetical parameter estimation from sequencing data. Bioinformatics 27, 2987-2993 (2011).

41. S. Greenblum, R. Carr, E. Borenstein, Extensive strain-level copy-number variation across human gut microbiome species. Cell 160, 583-594 (2015).

42. O. Reyes et al., 'Integron'-bearing vectors: a method suitable for stable chromosomal integration in highly restrictive corynebacteria. Gene 107, 61-68 (1991).

Acknowledgments: We are deeply grateful to the participants who provided sebaceous samples from their skin hair follicles. Funding: R01 AI101018 (KPL and MAF), U41 AT008718 (RGL), NSERC Discovery RGPIN-2016-03962 (RGL), NIH grants DP1 DK113598 (MAF), R01 DK110174 (MAF); an HHMI-Simons Faculty Scholars Award (MAF), an Investigators in the Pathogenesis of Infectious Disease award from the Burroughs Wellcome Foundation (MAF), JC is supported by Seed Funding from the Cleveland Clinic Foundation. Authors contributions: Conceptualization: JC, MAF, KPL. Investigation: JC, JBS, SFR, KLK, ALB, AAA, AVM, 
WRW, SW, RDH, MSD, RGL. Figures: JC, JBS, SFR, KLK, ALB, AAA, MAF. Writing of the original draft: JC, SFR, MAF, KPL. Writing, review and editing: JC, SFR, KLK, ALB, AAA, PCD, HHK, JAS, RGL, MAF, KPL. Competing interests: none declared. Data and materials availability: All data is available in the main text or the supplementary materials.

\section{Supplementary Materials:}

Materials and Methods

Figures S1-S18

Tables S1-S9 


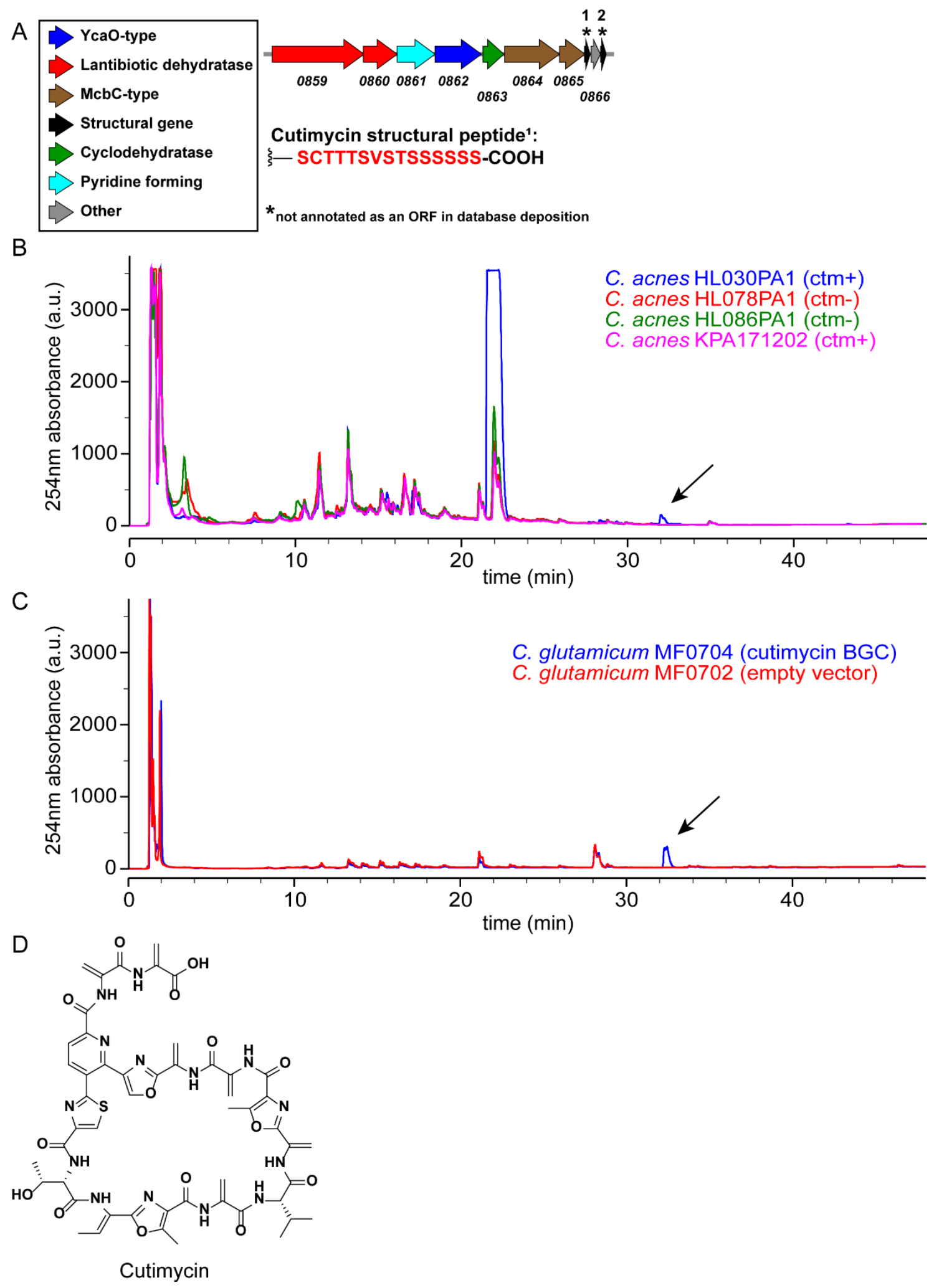

Fig. 1. Detection of cutimycin (ctm), the product of BGC ppa0859-0866, in native and heterologous hosts. A) Arrow representation of the ppa0859-0866 BGC from C. acnes KPA171202. B) HPLC profiles for crude ethyl acetate extracts of select ppa0859-0866 BGC+ 
and - C. acnes strains. The thiopeptide product of ppa0859-0866, dubbed cutimycin, elutes at $73.5 \% \mathrm{MeCN}$ as visible in the blue trace from $C$. acnes HL030PA1. Arrow indicates the cutimycin peak. C) Comparison of HPLC profiles of crude cell extracts of $C$. glutamicum hosting the cutimycin BGC on a plasmid (blue) versus hosting the empty vector control (red). Arrow indicates the cutimycin peak. D) Structure of the Cutibacterium-produced thiopeptide cutimycin.

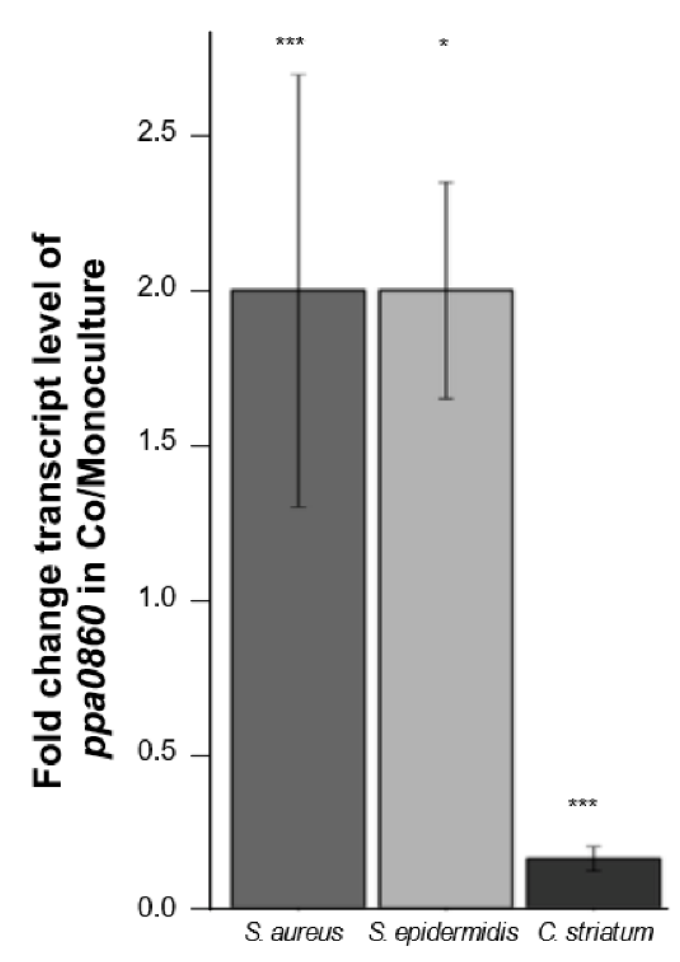

Fig. 2. The $C$. acnes ppa0860 transcript increases during cocultivation with Staphylococcus. The ratio of qRT-PCR results for ppa0860 from co- vs. monoculture with $S$. aureus (Sau, n=8, $p=0.0001)$, S. epidermidis (Sep, $\mathrm{n}=3, p=0.047)$ and $C$. striatum $(C s t, \mathrm{n}=3, p=0.00005)$. Paired ttest. Error bars are SD. 

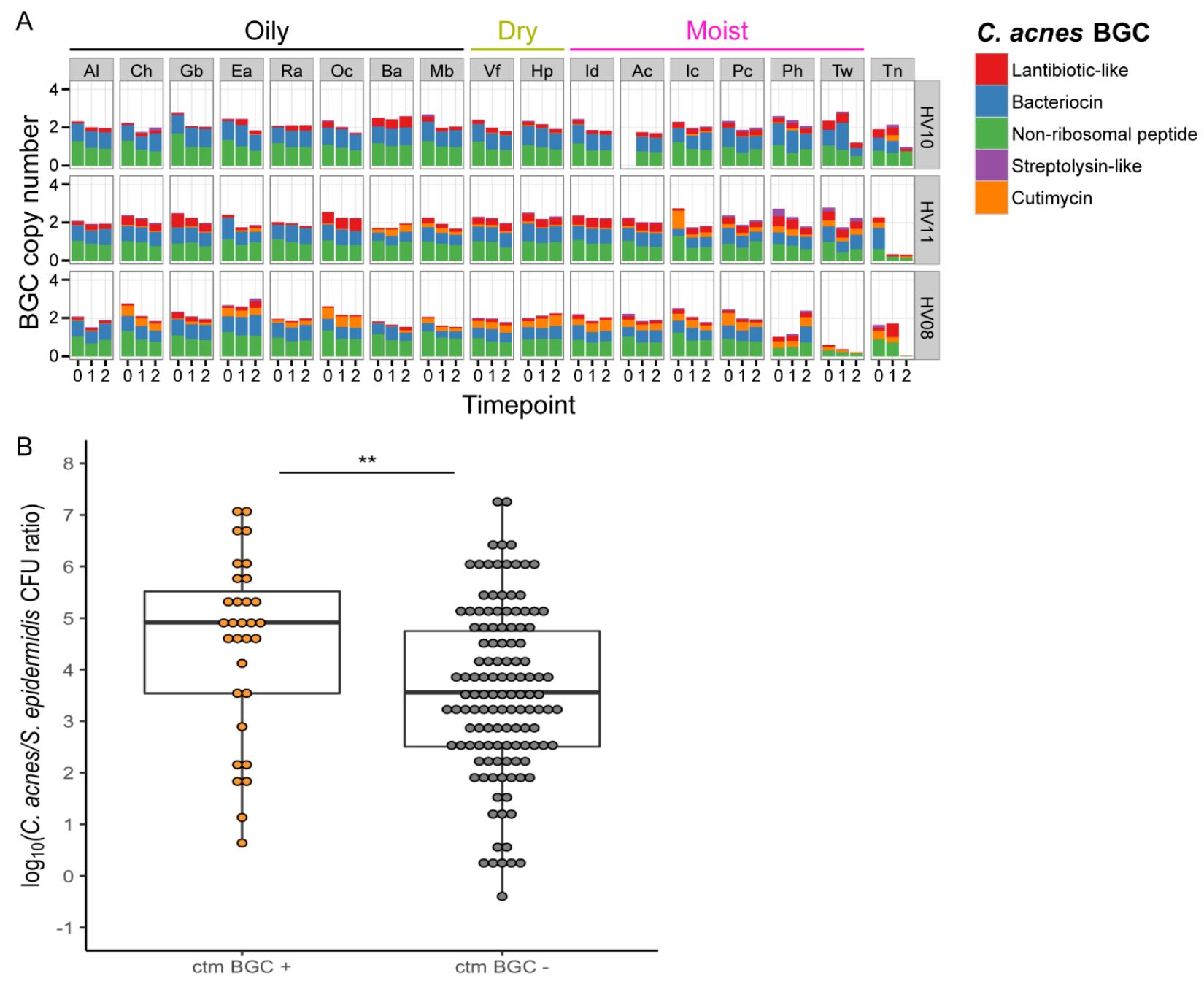

Fig 3. A) Example of spatial and temporal distribution of C. acnes BGCs in the skin metagenomes of three healthy individuals with low, medium or high relative abundance of the cutimycin BGC across 18 skin sites. Rows group samples from a single volunteer (coded Healthy Volunteer (HV) 01-12), columns represent samples across a specific body site (coded Ac through Vf). Cells contain bar graphs for each of the three time points, depicting the copy number of the $C$. acnes BGCs, which are standardized by comparing against $13 C$. acnes housekeeping genes. The BGC types, bacteriocin, lantibiotic, non-ribosomal peptide, streptolysin-like peptide and cutimycin are color coded and their abundances are stacked on top of each other (a colored bar with a height of 1 means all of the $C$. acnes in the sample harbor that $\mathrm{BGC}$, whereas a height of 0 means the $\mathrm{BGC}$ is absent in all of the $C$. acnes $) . \mathrm{Al}=$ alar crease, $\mathrm{Ch}$ $=$ cheek, $\mathrm{Gb}=$ glabella, Ea = external auditory canal, $\mathrm{Ra}=$ retroauricular crease, Oc = occiput, $\mathrm{Ba}=$ back, $\mathrm{Mb}=$ manubrium, $\mathrm{Vf}=$ volar forearm, $\mathrm{Hp}=$ hypothenar palm, $\mathrm{Id}=$ interdigital web space, $\mathrm{Ac}=$ antecubital fossa, $\mathrm{Ic}=$ inguinal crease, $\mathrm{Pc}=$ popliteal fossa, $\mathrm{Ph}=$ plantar heel, $\mathrm{Tw}=$ toe web space, $\mathrm{Tn}=$ toenail (see also diagram in Fig. S16A). B) Box plots of the impact of the cutimycin (ctm) BGC presence (+) or absence (-) on the $\log _{10}$ ratio of $C$. acnes/S. epidermidis CFUs from individual human skin follicular plugs ( $\mathrm{n}=156$, each follicle is represented by a dot) collected from 16 participants in total. For statistical analysis, data were pooled based on the assumption that follicles within an individual are independent. There was a statistically 
bioRxiv preprint doi: https://doi.org/10.1101/594010; this version posted March 30, 2019. The copyright holder for this preprint (which was not certified by peer review) is the author/funder. All rights reserved. No reuse allowed without permission.

significant difference in Cac/Sep (Wilcoxon signed-rank test, $p=0.006$ ) between ctm + and ctmsamples. 